\title{
AN OVERVIEW OF THE NEW CLIC TEST FACILITY (CTF3)
}

\author{
R.Corsini for the CTF3 Study Team, CERN, Geneva, Switzerland
}

\begin{abstract}
The CLIC (Compact Linear Collider) RF power source is based on a new scheme of electron pulse compression and bunch frequency multiplication, in which the drive beam time structure is obtained by the combination of electron bunch trains in isochronous rings. The next CLIC Test Facility (CTF3) at CERN will be built in order to demonstrate the technical feasibility of the scheme. It will also constitute a $30 \mathrm{GHz}$ source with the CLIC nominal peak power and pulse length, for RF component testing. CTF3 will be installed in the area of the present LEP preinjector (LPI) and its construction and commissioning will proceed in stages over five years. In this paper we present an overview of the facility and provide a description of the different components.
\end{abstract}

\section{INTRODUCTION}

The CLIC design of an $\mathrm{e}^{+} \mathrm{e}^{-}$linear collider aims at a centre-of-mass energy in the multi $\mathrm{TeV}$ range. Its main characteristics are the choice of acceleration with high gradients $(150 \mathrm{MV} / \mathrm{m})$ in high frequency $(30 \mathrm{GHz})$ normal-conducting structures. The design power is 240 MW per meter of linac, with a pulse length of 140 ns. A very efficient and reliable RF source is required, in a frequency region above the usual range of conventional sources, such as klystrons. A two-beam acceleration scheme is proposed [1], in which a high-current drive beam runs parallel to the high-energy main beam. The drive beam time structure ( $2 \mathrm{~cm}$ of bunch spacing) has a strong $30 \mathrm{GHz}$ component and the RF power is extracted from it periodically in resonant structures and transferred to the main beam. The drive beam is initially accelerated using cavities operating at low RF frequency, where commercial power sources are available. This acceleration takes place in fully-loaded cavities, so that all the RF power is converted into beam energy. The drive beam bunch trains are then interleaved by injection with transverse RF deflectors in isochronous rings, thereby increasing the bunch repetition frequency and shortening the bunch train.

The main goal of CTF3 is to demonstrate the key concepts of the new RF power generation scheme, namely the bunch combination scheme and the fully-loaded accelerator operation. The drive beam pulse obtained after combination (140 ns, $35 \mathrm{~A})$ will be sent to special resonant structures to produce $30 \mathrm{GHz}$ RF power with the nominal CLIC parameters, to test accelerating cavities and waveguide components.

The project is based in the PS Division of CERN, with collaboration from other Divisions, as well as from INFNFrascati, IN2P3/LAL at Orsay and SLAC. The facility will be built in the existing LPI complex and will make maximum use of equipment available after the end of LEP operation. In particular, the existing RF power plant at 3 $\mathrm{GHz}$ from the LEP injector Linac (LIL) and most of the LPI magnets will be used.

CTF3 will be built in stages over five years. The new accelerating structures, adapted to high current operation, will not be available before 2003. Therefore, it is planned at first to perform a low current test (CTF3 Preliminary Phase), using the present LIL structures [2]. The experimental program of this phase will start in Autumn 2001 with the goal to demonstrate the electron beam combination scheme. Since the beam current will be limited, the $30 \mathrm{GHz}$ RF power production and the study of collective effects will only be possible in later phases. As the new hardware becomes available, it will be installed in the LPI complex. A second stage (CTF3 Initial Phase), using the new linac, will allow for a test of fully- loaded acceleration and will have a limited power production capability. The final configuration of CTF3 will be reached in the third stage (CTF3 Nominal Phase, see Figure 1) and is described below.

\section{CTF3 DESCRIPTION}

\subsection{Drive Beam Injector}

The drive beam injector [3] is to be built in collaboration with SLAC (providing the gun triode and the beam dynamics design) and LAL/Orsay, (providing the gun electronics circuitry and the $3 \mathrm{GHz}$ pre-bunchers).

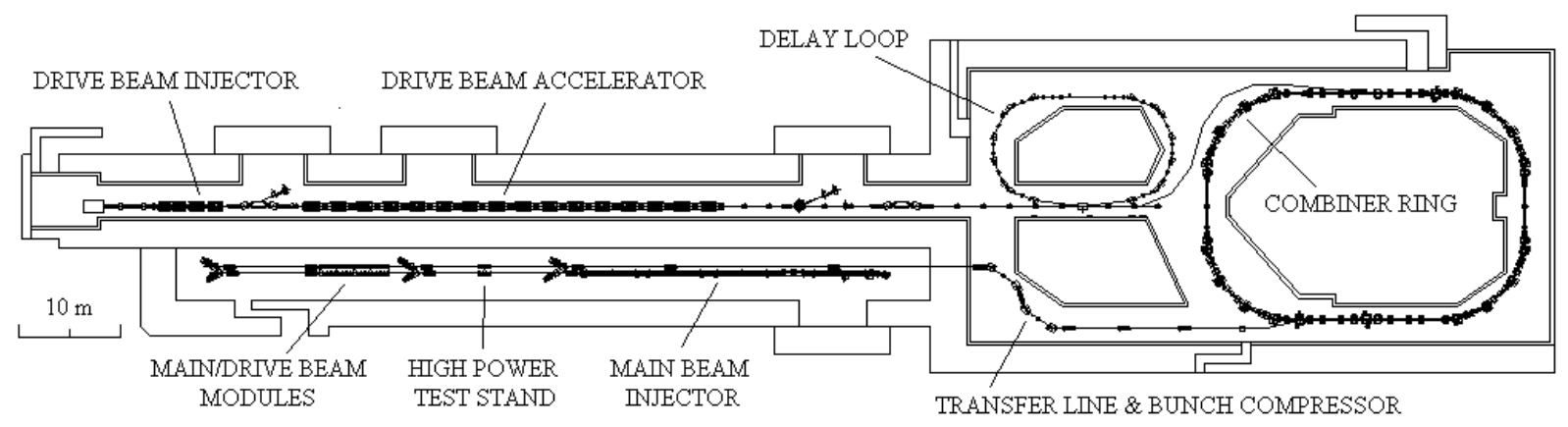

Figure 1: Layout of the final configuration of CTF3 (Nominal Phase). 
The $1.6 \mu$ s long drive beam pulse is generated by a 140 $\mathrm{kV}, 9 \mathrm{~A}$ thermionic triode gun. The gun is followed by a bunching system composed of a set of $1.5 \mathrm{GHz}$ subharmonic bunchers, a $3 \mathrm{GHz}$ pre-buncher and a $3 \mathrm{GHz}$ graded- $\beta$ travelling-wave buncher. The phase of the subharmonic bunchers is switched rapidly by $180^{\circ}$ every $140 \mathrm{~ns}$. In order to obtain a fast switching time ( $\leq 4 \mathrm{~ns})$, the $1.5 \mathrm{GHz}$ RF source must have a broad bandwidth (about $10 \%$ ) and a peak power of $500 \mathrm{~kW} \mathrm{[4].} \mathrm{The}$ bunches thus obtained are spaced by $20 \mathrm{~cm}$ (two $3 \mathrm{GHz}$ buckets) and have a charge of $2.3 \mathrm{nC}$ each, corresponding to an average current of $3.5 \mathrm{~A}$. As a result of the phase switching, the drive beam pulse is composed of $140 \mathrm{~ns}$ sub-pulses, which are phase-coded and can be later separated by transverse deflectors working at $1.5 \mathrm{GHz}$.

The injector is completed by two $3 \mathrm{GHz}$ travelling wave structures, bringing the beam energy up to about $20 \mathrm{MeV}$. Solenoidal focusing with a maximum on-axis field of $0.2 \mathrm{~T}$ is used all along. A magnetic chicane with collimators downstream of the injector will eliminate the low energy beam tails produced by the bunching process. The chicane region will also be instrumented to perform emittance and energy-spectrum measurements. An alternative option to the thermionic injector scheme, based on the use of an RF photo-injector, is also under study as a potential later upgrade [5].

\subsection{Drive Beam Accelerator}

The drive beam pulse is brought to its final energy $(150 \mathrm{MeV})$ in the drive beam accelerator, composed of 8 modules of $4.5 \mathrm{~m}$ length. Each module consists of two accelerating structures, identical to the ones used in the injector, a beam position monitor, a quadrupole triplet and a pair of steering magnets. Beam simulations have shown that the initial value of the normalised rms emittance ( $100 \pi \mathrm{mm} \mathrm{mrad}$ ) is conserved during acceleration despite the high beam current and the long beam pulse, provided that the transverse Higher Order Modes (HOMs) are suppressed. The travelling wave structures work in the $2 \pi / 3$ mode, have an active length of $1.13 \mathrm{~m}$ with a filling time of $100 \mathrm{~ns}$ and operate at a loaded gradient (nominal beam current) of about $8 \mathrm{MV} / \mathrm{m}$, with an RF-to-beam efficiency of $97 \%$. Two different structure designs have been developed to obtain the HOM suppression, and are presently being compared. The first is derived from the 30 $\mathrm{GHz}$ Tapered Damped Structure (TDS) of the CLIC main linac [6], using four waveguides with wide-band $\mathrm{SiC}$ loads in each accelerating cell. The waveguides act as a high-pass-band filter, since their cut-off frequency is above the fundamental frequency but below the HOM frequency span. The $Q$-value of the first dipole is thus reduced to about 18. A further reduction of the long range wake-fields is achieved by a spread of the HOM frequencies along the structure (detuning), obtained by varying the aperture from $34 \mathrm{~mm}$ to $26.6 \mathrm{~mm}$. A full prototype of this structure has been built and power tested up to $40 \mathrm{MW}$. The second approach (called SICA, for Slotted Iris Constant Aperture) uses four radial slots in the iris to couple the HOMs to SiC loads (see Figure 2). In this approach the selection of the modes coupled to the loads is not made by frequency discrimination but is obtained through the field distribution of the modes, so that all dipole modes are damped. The $Q$-value of the first dipole is reduced to about 5. The HOM detuning is obtained in this case by nose cones of variable geometry. The aperture can therefore be kept constant at $34 \mathrm{~mm}$, yielding a smaller amplitude of the short-range wakefield. A SICA test prototype is under construction. The RF power is supplied by eight $30 \mathrm{MW}$ klystrons and compressed by a factor 2 to give a peak power at each structure input of about $30 \mathrm{MW}$. The pulse compression system uses a programmed phase ramp to get an almost rectangular RF pulse [7]. A very good amplitude and phase stability on the RF pulse flat top is required to minimise the energy spread along the drive beam pulse.

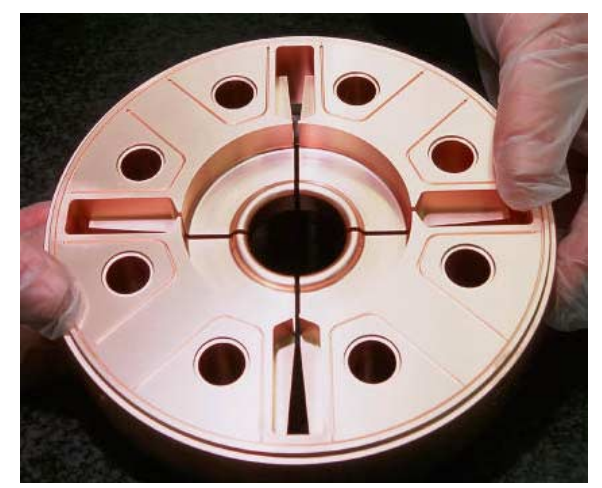

Figure 2: Prototype cell for the SICA structure.

\subsection{Delay Loop and Combiner Ring}

After the linac, a first stage of electron pulse compression and bunch frequency multiplication of the drive beam is obtained using a transverse RF deflector at $1.5 \mathrm{GHz}$ and a $42 \mathrm{~m}$ delay loop. The phase-coded subpulses are first separated and then recombined by the deflector after half of them have been delayed in the loop. The beam is then a sequence of five $140 \mathrm{~ns}$ long pulses with twice the initial current, separated by 140 ns "holes" (see Figure 3). An $84 \mathrm{~m}$ long combiner ring is then used for a further stage of pulse compression and frequency multiplication by a factor five, through injection with $3 \mathrm{GHz}$ transverse RF deflectors [1].

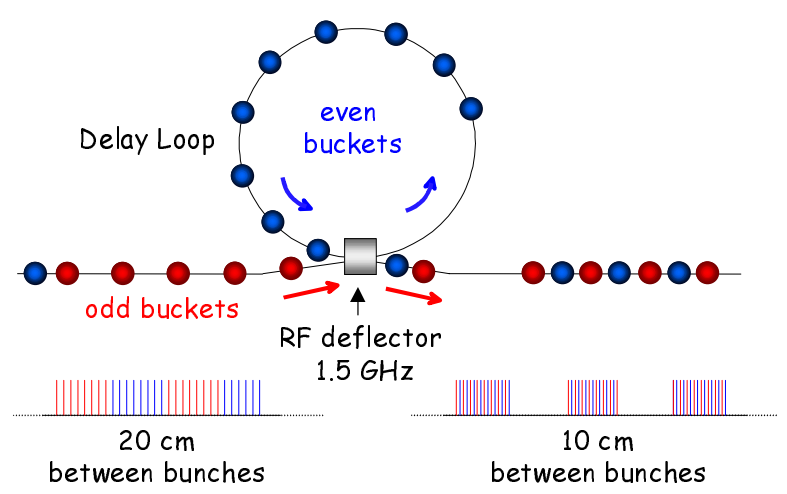

Figure 3: Schematic description of the pulse compression and frequency multiplication process using a delay loop and a transverse RF deflector. 
After the combiner ring, a single 140 ns long drive beam pulse with a current of $35 \mathrm{~A}$ is obtained. The final bunch spacing is $2 \mathrm{~cm}$.

The design of the delay loop, the combiner ring and the related beam lines is under the responsibility of INFN/Frascati [8]. Both the delay loop and the ring must be isochronous in order to preserve the short bunch length required. The ring consists of four isochronous arcs, two short straight sections and two long straight sections for injection and extraction. The ring arcs are triple-bend achromats, with negative dispersion in the central dipole. A precise tuning of the momentum compaction value can be obtained by a variation of the magnetic strength of the central quadrupole in each arc. Three sextupole families in the arcs provide a cancellation of the second-order momentum compaction, while controlling both the horizontal and vertical chromaticities. Two wiggler magnets are foreseen in order to adjust the ring circumference precisely to a $\mathrm{N}+1 / 5$ multiple of the bunch spacing (with a total adjustment range of $\pm 1 \mathrm{~mm}$ ), and are placed in the short straight sections (one similar wiggler is located in the delay loop). Prototypes of these wigglers are under construction. The injection and extraction regions, with similar requirements, have identical lattices. Injection and extraction septa are placed symmetrically in the centre of the long straight sections. The $\pi$ betatron phase advance between the two injection deflectors is obtained by four quadrupoles arranged symmetrically around the septum. In the extraction region this assures the required $\pi / 2$ phase advance between the extraction kicker and the septum.

A potential problem of the combination process with a high bunch charge is the effect of multi-bunch beam loading on the fundamental mode of the deflecting cavities. Detailed studies of the effect have been made [9], showing that the transverse beam stability can be sufficiently improved by a proper choice of the deflector parameters, of the $\beta$-function at injection and of the ring tune. The results are summarised in Figure 4.

The use of short, high-charge bunches also puts stringent requirements on the ring impedance and makes coherent synchrotron emission a serious issue. The main effects are beam energy loss and energy spread increase.

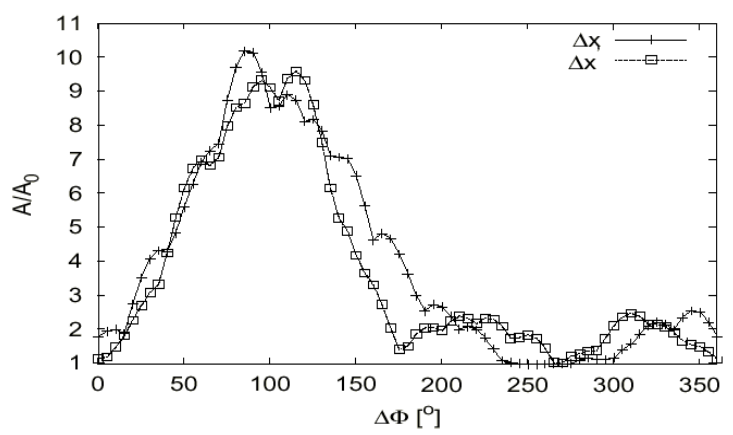

Figure 4: Amplification of an initial error in position and angle of the injected beam as a function of the betatron phase advance in the ring, after five turns. The ring tune has been chosen in the low amplification region, close to a phase advance of $260^{\circ}$.
In order to minimise these effects, the rms bunch length can be increased from its value of $1.3 \mathrm{~mm}$ in the linac to a maximum of $2.5 \mathrm{~mm}$ in the delay loop and ring, by a magnetic chicane placed at the end of the linac. After combination, the individual bunches are then compressed in length to about $0.5 \mathrm{~mm} \mathrm{rms}$ in a magnetic bunch compressor. The drive beam pulse is then transported to the $30 \mathrm{GHz}$ test area.

\subsection{Main Beam and $30 \mathrm{GHz}$ Test Area}

A single $30 \mathrm{GHz}$ decelerating structure, optimised for maximum power production, will be used in a high power test stand where CLIC prototype accelerating structures and RF components can be tested at nominal power and beyond. Alternatively, the drive beam can be used in a string of decelerating structures to power a representative section of the CLIC main linac and to accelerate a probe beam. The probe beam is generated in a $3 \mathrm{GHz}$ RF photoinjector and pre-accelerated above $100 \mathrm{MeV}$ using $3 \mathrm{GHz}$ accelerating structures recuperated from LIL. It can be accelerated further to about $500 \mathrm{MeV}$ in the $30 \mathrm{GHz}$ CLIC accelerating structures powered by the drive beam, operated at a maximum gradient of $150 \mathrm{MV} / \mathrm{m}$. This setup will allow us to simulate realistic operating conditions for the main building blocks of the CLIC linac.

\section{REFERENCES}

[1] R. Corsini (ed.) and 15 co-authors, "The CLIC RF Power Source - A Novel Scheme of Two-Beam Acceleration for $\mathrm{e}^{ \pm}$Linear Colliders", CERN 99-06 (1999).

[2] R. Corsini, A. Ferrari, L. Rinolfi, T. Risselada, P. Royer, F. Tecker, "Beam Dynamics for the Preliminary Phase of the New CLIC Test Facility (CTF3)", this conference.

[3] H. Braun, R. Pittin, L. Rinolfi, F. Zhou, B. Mouton, R. Miller, D. Yeremian, "An Injector for the CLIC Test Facility (CTF3)", CERN/PS 2000-052, Proc. of the XX Linac Conference, August 21-25, 2000, Monterey, USA.

[4] G. Phillips, "A 500 kW L-Band Klystron with Broad Bandwidth for Sub-Harmonic Bunching in the CLIC Test Facility", Proc. of the $5^{\text {th }}$ Modulator-Klystron Workshop for Future Linear Colliders, April 26-27, 2001, Geneva, Switzerland.

[5] I.N. Ross, "Feasibility Study for the CERN CLIC PhotoInjector Laser System”, CLIC Note 462 (2000).

[6] M. Dehler, I. Wilson, W. Wuensch, "A Tapered Damped Accelerating Structure for CLIC", CERN/PS 98-040 (LP), Proc. of the XIX Linac Conference, August 23-28, 1998, Chicago, USA.

[7] I. Syratchev, "RF Pulse Compressor Systems for CTF3", Proc. of the $5^{\text {th }}$ Modulator-Klystron Workshop for Future Linear Colliders, April 26-27, 2001, Geneva, Switzerland.

[8] C. Biscari and 12 co-authors, "CTF3 - Design of Driving Beam Combiner Ring", Proc. of the $7^{\text {th }}$ European Particle Accelerator Conference, June 26-30, 2000, Vienna, Austria and CLIC Note 471 (2001).

[9] D. Alesini, R. Corsini, A. Gallo, F. Marcellini, D. Schulte, I. Syratchev, "Studies on the RF Deflectors for CTF3", Proc. of the $7^{\text {th }}$ European Particle Accelerator Conference, June 26-30, 2000, Vienna, Austria and CLIC Note 472 (2001). 